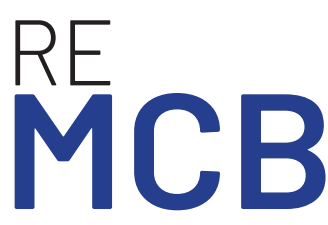

Revista Ecuatoriana de Medicina y Ciencias Biológicas

Volumen 42. No. 2, Nov 2021

\section{Inmunotipificación y cuantificación de cadenas ligeras libres en pacientes ecuatorianos con gammapatías monoclonales}

\author{
Immunotyping and quantification of free ligh chains in Ecuadorian patients \\ with monoclonal gammopathy
}

\author{
Diego Talledo Pinargote ${ }^{1,6}$, Aron Zamora Cedeño², Lilian Sosa Fernández-Aballiß ${ }^{3}$ \\ Gusdanis Alberto Campos García y I Ivon Howland Álvarez ${ }^{5 *}$
}

Resumen: Actualmente, algunas de las neoplasias hematológicas, entre ellas, las gammapatías monoclonales (GM) son incurables a pesar de los avances en las terapias oncológicas. Los estudios de GM en Ecuador son escasos; en las últimas estadísticas, se muestra que el mieloma múltiple (MM) se encuentra entre los primeros 20 tumores en el país. Las pruebas de diagnóstico más utilizadas son electroforesis y cadenas ligeras. Con el objetivo de caracterizar los resultados en suero de electroforesis de proteínas (ELP), inmunotipificación (IT) y cuantificación de cadenas ligeras libres en sangre (CLL) de pacientes con GM en Portoviejo, Manabí, Ecuador, se realizó un estudio observacional, descriptivo y retrospectivo que incluyó 237 ELP, 42 IT y 58 CLL, provenientes de pacientes atendidos en Servicios de Hematología de la región desde enero a julio 2019. Se utilizaron las técnicas de Sebia ELP e IT y para CLL la de Seralite. El 14\% de las ELP fueron GM, 70\% de género masculino con un promedio de 64 años. Más de la mitad de las GM tenían diagnóstico de MM, de los cuales 15\% eran menores de 40 años. EL 69\% de las IT fueron positivas y predominó el isotipo lgG kappa (K). El 91\% de las CLL presentaron patológica la relación $\mathrm{k} / \lambda$. La IT no mostró utilidad diagnóstica en los casos de MM no secretor u oligosecretor. Los resultados de CLL fueron significativamente mayores a las reportados por la técnica de cadenas ligeras totales, por lo que esta prueba aumentó la posibilidad de detección de MM en todas sus variantes.

Palabras claves: gammapatía monoclonal, componente monoclonal, electroforesis de proteína, inmunotipificación, cadenas ligeras libres.

Abstract.- Despite advances in oncological therapies, certain hematological neoplasms, among them monoclonal gammopathies (MG), are at present incurable. Studies of MG in Ecuador are scarce; the latest statistics show that multiple myeloma (MM) is among the top 20 most frequent types of tumors in the country. To characterize the results of immunotyping (IT) and quantification of free light chains in the blood (FLC) of patients with MG in Portoviejo, Manabí Ecuador, an observational, descriptive, longitudinal and retrospective study was carried out that included 237 protein electrophoresis (PEL), 42 IT and 58 FLC, from patients seen at the regional hematology services from January to July 2019. The techniques were used Sebia ELP and IT and for CLL, Seralite. Of the GM studied, 14\% were GM; $70 \%$ male with an average age of 64 years and a range of 31-87 years. More than half of the GM had a diagnosis of Multiple Myeloma (MM), of which $15 \%$ were under 40 years of age. For IT, 69\% were positive and the IgG kappa (K) isotype predominated. IT did not show diagnostic utility in non-secretory or oligosecretory MM cases. A total of $91 \%$ of the FLC presented pathological results of a $\mathrm{k} / \lambda$ relationship. The FLC results were significantly higher than those reported by the technique traditionally used in the total light chain region, meaning that the test increased the possibility of MM detection of in all its variants.

Keywords: monoclonal gammopathy, monoclonal component, protein electrophoresis, immunotyping, free light chains 


\section{Introducción}

Las gammapatías monoclonales (GM) son un grupo de trastornos caracterizados por la proliferación de un clon de células plasmáticas, proveniente de los linfocitos B que, generalmente, producen en exceso, y de manera descontrolada, inmunoglobulinas idénticas, subunidades o fragmentos idénticos entre sí; se las denomina componente/proteína monoclonal o proteína M (CM) (Laribi et al. 2018; Murata et al. 2019).

Por lo general, una GM puede corresponder a inmunoglobulina intacta secretada en exceso, un exceso exclusivo de cadenas ligeras producidas sin una cadena pesada asociada, o ambas. (Marco 2014; Caravaca-Fontán et al. 2017; Delgado 2019). Las GM más mencionadas incluyen: GM de Significado Incierto (GMSI), Mieloma Múltiple (MM) y sus variantes (Morrison et al. 2019) y Amiloidosis primaria (Murata et al. 2019; Seth et al. 2020). Cualquiera que sea el caso, la evaluación del CM es considerada el marcador tumoral por excelencia; por lo que su estudio es fundamental para el diagnóstico certero, pronóstico y seguimiento de la enfermedad (Marco 2014; Caravaca-Fontán et al. 2017; Delgado 2019).

Actualmente, algunas de las neoplasias hematológicas, entre ellas, las GM son incurables a pesar de los avances en las terapias oncológicas. Una de las descritas con mayor frecuencia es el MM que, según la Organización Mundial de la Salud (OMS), representa el 1\% de todas las neoplasias y alrededor del 10-15\% de las neoplasias hematológicas. Es una enfermedad maligna y multisistémica, que se ha descrito con más frecuencia en la población afrodescendiente y, en la cual, la presencia del CM se puede identificar por diversas técnicas de laboratorio clínico tanto en sangre como en orina (Rincón 2016).

En América Latina, resaltan las investigaciones epidemiológicas procedentes de países como Argentina, Brasil, Chile y México, las cuales han documentado información clínica y genética del MM (Montesdeoca Narváez 2015). Los estudios del MM en Ecuador son escasos y se debe estudiar su comportamiento por varias causas, incluyendo que la población ecuatoriana es una mezcla compleja entre nativos americanos, europeos y africanos. En un estudio genético de 61 pacientes con MM, realizado por Leone et al. (2017), los cuales fueron comparados con un control saludable, se mencionó que en la ascendencia genética de individuos mestizos ecuatorianos con MM, la ascendencia africana no influyó en el desarrollo de este cáncer. Se planteó, además, que los casos con mayor porcentaje de ascendencia nativa americana tuvieron más riesgo de desarrollar el MM. El grupo étnico mestizo, con todos sus componentes, nativo americano, europeo y africano, tuvo su propio comportamiento con respecto al MM. Esto explicaría las diferencias en los datos clínicos en relación con otras poblaciones y hace que la población ecuatoriana tenga sus particularidades genéticas que la diferencian de las de otras regiones del mundo para este tipo de cáncer.

El Registro Nacional de Tumores (RNT) de la Sociedad de Lucha contra el Cáncer (SOLCA) en Ecuador, en las estadísticas publicadas en el año 2019, mostró que el MM se encuentra entre los primeros 20 tumores en el país, registrando un 55\% de casos en hombres, un $45 \%$ de casos en mujeres (Cueva et al. 2019). En el RNT de 2013 se indicó que el 14\% de los casos con MM tenían relación con el curtido de cuero y agricultura y que, en rasgos generales, desde 1986 han aumentado el número de casos de MM en el país.

Basados en el hecho de que era difícil el diagnóstico preventivo y la enfermedad era detectada en estadios en los cuales había compromiso sistémico, en 1975 se desarrolló el sistema de estratificación del MM de Durie-Salmon, el cual une los principales parámetros clínicos en correlación con la masa de células de mieloma cuantificados y con énfasis en los rangos clínicos de cada paciente de manera individual. En 2009, el Grupo Internacional de Trabajo en Mieloma (IMWG, del inglés International Myeloma Working Group) estableció las primeras normas publicadas para el diagnóstico del MM mediante criterios diferidos. Desde entonces, el IMGW publica las directrices para el análisis en el diagnóstico y seguimiento del MM, además de las pruebas de laboratorio tradicionales; mismas que, conjunto a las técnicas de imagen, ayudan a clasificar mejor las opciones de tratamiento, evaluar la extensión de la enfermedad y estadificar el MM (Alvarado-lbarra et al. 2015). 
Los criterios actualizados representan un cambio de paradigma ya que permiten el diagnóstico temprano y la iniciación de la terapia antes del daño al órgano terminal. Cuando se sospecha clínicamente de MM en los pacientes, se debe analizar la presencia de CM utilizando una combinación de pruebas que deben incluir una electroforesis de proteína en suero (ELP), inmunofijación y el ensayo de cadenas ligeras libres en suero (CLL) (Rajkumar 2018). Paralelamente al desarrollo de la ELP capilar, también ha ido avanzando un método alternativo para identificar el CM: la llamada electroforesis de inmunotipificación por sustracción, también conocida por su término en inglés inmunotyping (IT). Esta técnica ha sido estandarizada desde 2008 y separa proteínas de suero tras una breve incubación del suero en la presencia de antisuero para cada una de las cadenas pesadas y ligeras, elimina los inmunocomplejos formados y permite la detección cualitativa de inmunoglobulinas monoclonales en función de su ausencia, en comparación con la electroforesis de proteínas séricas realizada sin antisueros. Asimismo, resulta de gran utilidad para la identificación de CM, pues, utiliza menor cantidad de muestra con un alto grado de resolución (McCudden et al. 2008) y tiene una sensibilidad muy aceptable; además, no utiliza colorantes, lo cual lo hace más inocua para el medio ambiente.

En este trabajo se estudiaron pacientes con GM diagnosticadas clínicamente y con detección electroforética del CM en ELP sérica, así como su confirmación mediante IT y CLL. Todo ello, para caracterizar los resultados de estas pruebas en el diagnóstico de los pacientes con GM en esta región y, de esta forma, concienciar al personal de salud de la necesidad de utilizarlas de forma rutinaria siempre que exista la sospecha diagnóstica de esta enfermedad.

\section{Materiales y métodos}

Se realizó un estudio observacional, descriptivo y retrospectivo desde enero a julio de 2019 con resultados electroforéticos del Laboratorio Clínico Gamma de Portoviejo. Se verificaron 237 ELP patológicas, 42 IT, 58 CLL y 58 cadenas ligeras totales (CLT) en pacientes a los que se les indicaron estas pruebas y en los que constaba el diagnóstico clínico del tipo de GM que presentaban. Para ello, se revisaron las historias clínicas de estos pacientes que fueron atendidos en el Servicio de Hematología del Hospital Oncológico "Dr. Julio Villacreses Colmont" (SOLCA) de la ciudad de Portoviejo.

Las IT y las ELP fueron resultados obtenidos con equipo Minicap Flex Piercing de Sebia. Para la cuantificación de CLL k y $\lambda$ se usó el dispositivo de flujo lateral inmunocromatográfico de SERALITE ${ }^{\circledR}$ y para su interpretación se empleó el lector ADxLR5 ${ }^{\circledR}$ Reader System de Abingdon. Se revisaron en las historias resultados para beta2 microglobulina, hemoglobina, creatinina y calcio. El procesamiento de los datos se realizó con el apoyo del programa SPSS (versión 23).

Se realizó la prueba de Wilcoxon para la comparación de los valores de relación $\mathrm{k} / \lambda$, en función de la prueba de laboratorio empleada (CLL y CLT), en los casos en los cuales ambos métodos fueron realizados a los mismos pacientes. Se empleó la prueba Mc Nemar para establecer si existían diferencias entre los resultados por las pruebas. En todos los casos, se consideró significativo un valor de $\mathrm{p}<0,05$.

El trabajo contó con la aprobación del Comité de Bioética de la Facultad de Ciencias de la Salud de la Universidad Técnica de Manabí, mismo que cuenta con los principios referentes al código de ética de acuerdo con la declaración de Helsinki. Toda la información de los pacientes contó con los principios de confidencialidad que rigen la investigación científica.

\section{Resultados}

En el período estudiado, del total de ELP realizadas, 81\% de los casos fueron patológicos y 19\% supuestamente normales. Según la interpretación del laboratorio clínico, al momento de analizar las alteraciones cualitativas en el proteinograma, se reportó que 29\% de los casos cursó con patrón inflamatorio crónico, 12\% con patrón inflamatorio agudo, 12\% con presencia de patrón anormal (disminución o aumento aislado de algunas de las fracciones no gammaglobulinas), 10\% con hipergammaglobulinemia o gammapatía policlonal, 3\% con hipogammaglobulinemia, $1 \%$ en el cual el puente beta-gamma fue clasificatorio para un patrón de enfermedad hepática, y 14\% presentó un patrón electroforético de GM. 
Del total de GM, 17\% presentó un patrón biclonal en la región gamma, lo cual correspondió al 3\% del total de las ELP patológicas. 70\% del total de las GM correspondió al género masculino y $30 \%$ al femenino. En cuanto a la edad, el promedio fue 64,5 años; el paciente con mayor edad fue de 87 años y el menor, de 31 años. El grupo etario con mayor número de GM correspondió a los mayores de 60 años, seguido del grupo que comprendió las edades entre 31-40 años, luego los de 51-60 años y, por último, el grupo de 41-50 años.

El 53\% de todas las GM detectadas se correspondió con el diagnóstico clínico de MM con características clásicas, 12\% fueron MM no secretor (MMNS), 14\% fueron linfomas (cuatro no Hodgkin, dos de ellos difuso y un caso de Hodgkin), 12\% correspondieron a un Síndrome mielodisplásico; 6\% tenían diagnóstico de Plasmocitoma solitario, 3\% GMSI y 3\% Leucemia linfoide crónica.

En cuanto a las IT, del total de GM detectadas, 69\% dieron positivas, es decir, se tipificó CM. Una única proteína monoclonal se observó en el 72\% de la IT positivas (figuras 1B y 2A), 21\% fueron GM biclonales (figura 2B) y 7\% componentes oligoclonales (figura 2C). Dentro de los CM únicos, el $67 \%$ fue $\lg$ K , $19 \% \lg G \lambda, 5 \% \operatorname{lgM}$ k (figura 3A) y 9\% se presentó en forma de cadena ligera de tipo $\lambda$ (figura 3B). En el caso de los 6 pacientes con biclonalidad, en dos pacientes los dos CM fueron lgG k, en dos se observó un CM lgG k y en uno lgM k, en un paciente cadenas pesadas y ligeras eran diferentes ( $\lg G \mathrm{~K}+\lg \mathrm{A} \lambda$ ) y hubo un paciente con dos cadenas ligeras de tipo $\mathrm{K}$.

La cuantificación de las inmunoglobulinas, por método nefelométrico, se relacionó con el tipo de CM identificado en los pacientes con MM. En el 61\% de los CM de tipo lgG, y el $67 \%$ de tipo IgM, se presentaron concentraciones de la inmunoglobulina implicada por encima de los rangos de referencia. Sin embargo, al analizar la inmunoglobulina implicada como CM, junto al descenso de las otras inmunoglobulinas, se observó que solo en el 33\% de los isotipos lgG existió disminución de la concentración de alguna de las otras dos lgA o lgM, ya sea en ambas o en, al menos, alguna de ellas.

Del total de las CLL realizadas, el 91\% de los resultados de pacientes diagnosticados con GM presentó resultados mayores a los valores de referencia en $k, \lambda$ o ambas. Para el resto, se reportaron valores dentro del rango de referencia. Al comparar los resultados de los valores del radio $k / \lambda$, por la técnica de $C L L$ que utiliza un suero policlonal específico para detectar epítopes de las cadenas ligeras libres $\mathrm{k}$ y $\lambda$ con los resultados obtenidos a partir del ensayo de CLT que cuantifica cadenas $\mathrm{k}$ y $\lambda$ unidas las inmunoglobulinas intactas, se observó que los resultados del radio $k / \lambda$ por la técnica CLL fueron significativamente mayores $(p=0,021 ; p<0,05)$ a los reportados por la prueba CLT.

Además, en los resultados encontrados de los pacientes con GM, se observó que el 59\% presentó valores de beta2 microglobulina mayores al rango de referencia y, en estos pacientes, también se presentaron valores elevados de las CLL. Es de destacar que, en las historias clínicas de los pacientes con MM, se observó que solamente un bajo porcentaje de ellos presentaron anemia (hombres 31\%, mujeres 12\%), creatinina sérica elevada (hombres 15\% y mujeres 12\%) y ninguno de ellos presento hipercalcemia.

\section{Discusión}

La inmunofijación en suero o su variante la IT consiste en la detección, mediante electroforesis y uso de anticuerpos, de proteínas monoclonales o CM. La IT está indicada en los pacientes que presentan un pico en las regiones beta o gamma de las ELP y que tienen sospecha de GM, especialmente, en el MM. De igual manera, si no es evidente la banda o pico monoclonal en la ELP, pero hay sospecha clínica de este tipo de neoplasia, está indicado la IT, ya que al poseer mayor sensibilidad y uso de anticuerpos específicos ayuda a identificar el CM. En la literatura se plantea que en el 93\% de los casos de pacientes con MM se detecta una proteína M en la inmunofijación y la cifra aumenta a $97 \%$ si se complementa con IF en orina. Por ello, se recomienda que la búsqueda del CM se realice tanto en suero como en orina. Aunque no cuantifica el componente monotípico, se emplea para el seguimiento y evaluación de respuesta al tratamiento de pacientes con estas neoplasias. 


\section{A $\quad$ B}

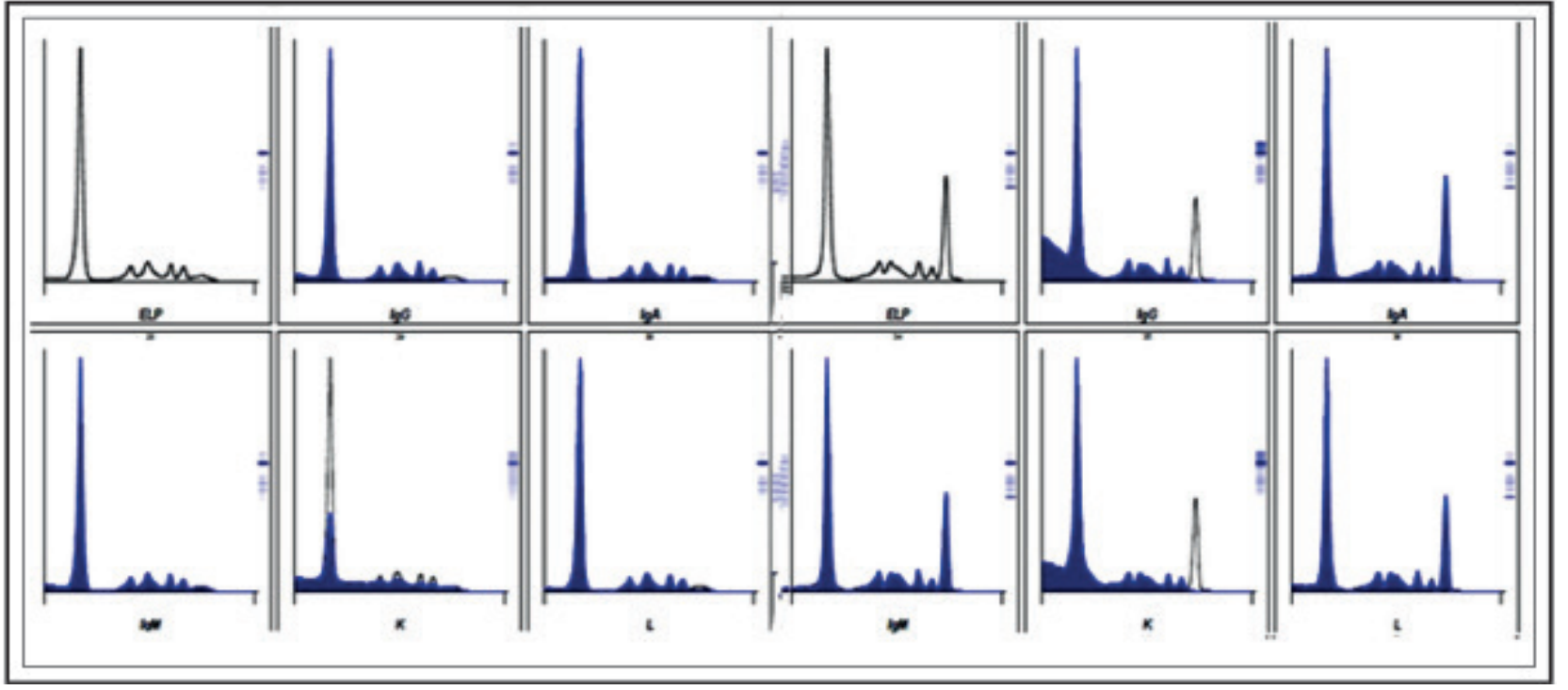

Figura 1. A: Inmunotipificación negativa; B: Inmunotipificación positiva para componente monoclonal lgG K.

Las IT fueron realizadas con la técnica Minicap Inmunotyping, basada en el principio de la electroforesis capilar en solución libre. En esta técnica, las proteínas son separadas y detectadas directamente durante la migración mediante absorbancia UV. Las inmunoglobulinas reaccionan específicamente con su antisuero correspondiente. Al resultado final del análisis, sobre cada perfil del antisuero ( $\lg G, \lg A, \lg M, k$ y $\lambda$ ), se superpone la curva de la ELP. La desaparición o sustracción en el perfil tratado con el antisuero en la fracción gamma o beta-gamma indica la presencia de una proteína monoclonal que, como se observa en la figura 1, muestra una IT negativa (A) donde no hay ninguna sustracción en los perfiles; en el otro (B), se muestra un resultado positivo donde es evidente la sustracción en los perfiles lgG y k, es decir, la IT es positiva para CM de tipo $\lg$ G K. Mediante la prueba de IT se pueden detectar concentraciones de CM de $150 \mathrm{mg} / \mathrm{L}$ a $500 \mathrm{mg} / \mathrm{L}$, pero no resulta de utilidad en el diagnóstico de MMNS, el cual se caracteriza por un patrón electroforético normal y una producción constante de concentraciones de cadenas livianas menores al límite de detección del tipo de prueba (Vázquez y Palma 2015).

Las gammapatías biclonales, por lo general, están relacionadas a discrasias de células plasmáticas; aunque, también, se pueden encontrar asociadas a otras neoplasias hematológicas, especialmente las linfoides de estirpe B, a enfermedades de tipo autoinmune o a crioglobulinemia (García-García et al. 2015). Las oligoclonales generalmente están asociadas con infecciones crónicas, enfermedades por inmunocomplejos, autoinmunes, hepatitis crónicas y, en algunos casos, con pacientes VIH positivos (Mexicana 2004).

El predominio del CM de tipo lgG de este trabajo guarda relación con otros estudios, los cuales indican una mayor cantidad de pacientes con MM de tipo lgG. En un estudio similar, publicado en el año 2013 en Cuba, se analizaron 285 pacientes con diagnóstico de MM, obteniendo como resultado que un 72,28\% presentaron MM de tipo IgG, 25,62\% MM de tipo IgA y 2,1 MM de tipo IgM (Arce-Hernández et al. 2013). Igualmente, en un estudio del Instituto Nacional de Enfermedades Neoplásicas de Lima, Perú, en el 2016, de una cohorte conformada por 92 pacientes diagnosticados con MM, el 59\% presentó CM de tipo lgG, 25\% IgA y el $13 \%$ tenían producción exclusiva de cadenas ligeras (Pizarro et al. 2016). En otra investigación, realizada en China, sobre la distribución de inmunotipado de paraproteína monoclonal sérica, entre agosto del 2013 a junio del 2015, se evaluaron 327 ELP, 46 casos correspondieron a ELP positivas. La distribución de inmunotipado más frecuente en suero fue $\lg \mathrm{G} k \operatorname{con} 25$ casos, seguida con $\lg A \lambda$ 7 casos, y e lgG $\lambda$ en sujetos con GM (Chang et al. 2016). 

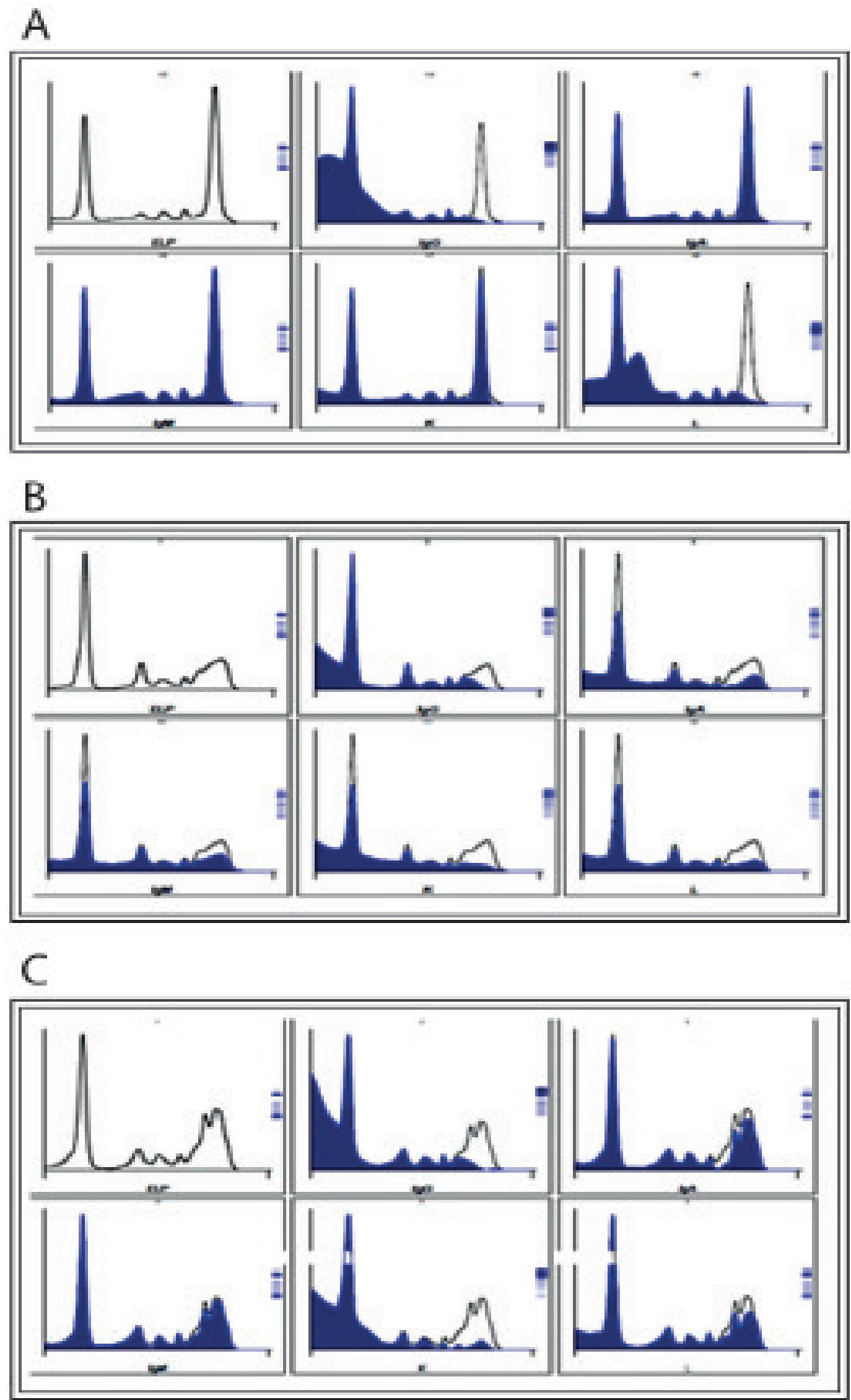

Figura 2. A: Inmunotipificación positiva IgG Lambda; B: Inmunotipificación positiva IgG Kappa-lgA Lambda; C: Inmunotipificación positiva oligoclonal 2 lgG Kappa-1 débil lgA Kappa. 


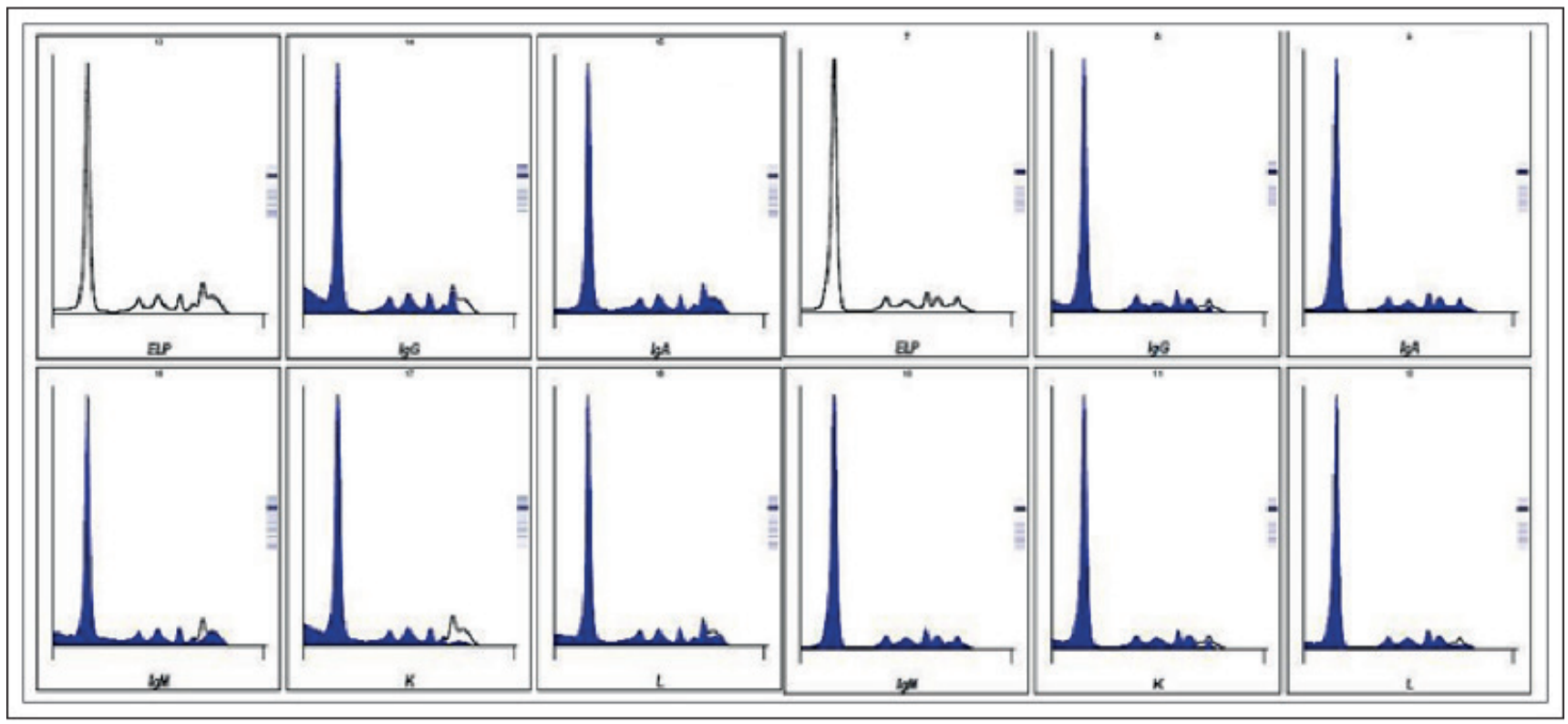

Figura 3. A: Inmunotipificación positiva IgM Kappa; B: Inmunotipificación positiva Lambda.

Durante varios años, los intentos de detección de CLL no tuvieron éxito, ya que ningún método analítico fue capaz de distinguir entre cadenas libres unidas a inmunoglobulina intacta y CLL. Sin embargo, a principios del año 2000, se desarrolló un ensayo capaz de detectar el epítopo oculto en la inmunoglobulina intacta y visible en la CLL. La determinación de estas cadenas libres es un marcador imprescindible para el diagnóstico y seguimiento de las GM. Existen inmunoensayos que cuantifican las cadenas $\mathrm{k}$ y $\lambda$, de manera automatizada, aportando gran especificidad y sensibilidad al diagnóstico de las GM. Desde el año 2009 hasta la actualidad, el IMWG recomienda la combinación de ELP, electroforesis por inmunofijación y CLL para el diagnóstico y seguimiento de las GM (Peña et al. 2018).

En varios estudios, se da a conocer la importancia del uso de CLL para la detección de monoclonalidad por su gran sensibilidad. En el año 2005, se identificaron 39 GM de un total de 1.003 muestras, con ayuda de técnicas de ELP; además, con ayuda de las CLL, se sumaron 16 pacientes aumentando la tasa de detección en un 43\% (Jenner 2014). Un estudio análogo en Chile reveló que, de un total de 312 pacientes estudiados, al incorporar el estudio de CLL, hubo un incremento del 29\% de sensibilidad (Peña et al. 2018). En el año 2016, en Perú, la determinación de las concentraciones de CLL y la evaluación del radio $k / \lambda$, fue útil en la detección de 81 del total de 92 pacientes con MM. Al analizar de manera conjunta los métodos de ELP, inmunofijación y CLL, se observó un incremento en la sensibilidad de detección, logrando un diagnóstico acertado del 100\% (Pizarro et al. 2016). En un estudio realizado en el 2017, sobre la validación clínica del ensayo de CLL de Seralite ${ }^{\circledR}$, un total de 576 pacientes se clasificaron como MM de cadenas livianas porque no tenían una proteína M intacta en la inmunofijación sérica; pero, sí tenían CLL en la inmunofijación de la orina. De estos pacientes, el 81\% también tenían una CLL detectable en suero por inmunofijación y la relación sérica $\mathrm{k} / \lambda$ fue anormal en todos los pacientes. Por lo tanto, pudieron diagnosticar con gran sensibilidad el mieloma de casos de cadenas livianas independientemente de los resultados en orina (Heaney et al. 2017).

En el 2009, el IMWG realizó un conjunto de recomendaciones acerca del esquema de diagnóstico inicial referido a la detección de la proteína monoclonal o CM. Esas guías son aceptadas y usadas en varios países, las cuales recomiendan la realización de una ELP junto con un ensayo de CLLs de la misma muestra y, si una o ambas dieran positivas, sobre la misma muestra se realiza una inmunofijación para tipificar la GM (Rajkumar et al. 2014). Además, según las directrices del IMWG para el análisis de CLLs en el MM y trastornos relacionados de los pacientes que tienen una enfermedad no secretora u oligosecretora y, para la mayoría de los pacientes con 
AL, los métodos tradicionales (ELP, IF y medición nefelométrica de cadenas pesadas) no son adecuados. El desarrollo del ensayo de CLLs ha demostrado su utilidad para el seguimiento de estos pacientes (Rajkumar et al. 2014), tal y como se muestra en los resultados de este trabajo.

\section{Conclusiones}

El 14\% de las electroforesis de proteínas séricas tenía diagnóstico de gammapatía monoclonal, $70 \%$ de ellas de pacientes del género masculino con un promedio de edad de 64 años. Más de la mitad de los pacientes con gammapatías monoclonales tenían diagnóstico de mieloma múltiple, de los cuales 15\% eran menores de 40 años. EL 69\% de las electroforesis por inmunotipificación séricas fueron positivas y predominó el isotipo lgG kappa (K). El 91\% de los resultados de cuantificación de cadenas ligeras libres en sangre fueron patológicos para el radio o relación $\mathrm{k} / \lambda$. La prueba de electroforesis por inmunotipificación sérica no mostró utilidad diagnóstica en los casos con diagnóstico clínico de mieloma múltiple no secretor u oligosecretor. Los resultados de cuantificación de cadenas ligeras libres en sangre fueron significativamente mayores a las reportados por la técnica de cuantificación de cadenas ligeras totales de la casa comercial Roche, ampliamente utilizada en el medio; es así que la cuantificación de cadenas ligeras o livianas libres aumentó la posibilidad de detección de mieloma múltiple en todas sus variantes con gran importancia en los de tipo oligosecretores.

\section{Agradecimientos}

A la dirección de Laboratorios Gamma de Portoviejo por facilitar datos para realizar este trabajo; a las autoridades de la Universidad Técnica de Manabí que, igualmente, proporcionaron facilidades para la obtención y el manejo de datos; a los médicos del Servicio de Oncohematología del Hospital de la SOLCA Portoviejo, que contribuyeron a la atención de los pacientes aportando datos sobre el comportamiento de la enfermedad; $y$, en especial, al Dr. Danilo Navarrete. A los pacientes, principales protagonistas de este trabajo y para los cuales están encaminados todos nuestros esfuerzos.

\section{Conflictos de interés}

Los autores declaran no tener conflictos de interés.

\section{Contribuciones de los autores}

DTP: Adquisición / Colección de datos, revisión bibliográfica, análisis e interpretación de datos, redacción de la primera versión del manuscrito, revisión del manuscrito.

AZC: Concepción y diseño del estudio, diseño experimental, análisis e interpretación de datos, redacción de la primera versión del manuscrito, revisión del manuscrito.

LSFA: análisis e interpretación estadística de datos, revisión del manuscrito.

ACG: Revisión bibliográfica, análisis e interpretación de datos, revisión del manuscrito, gestión del financiamiento.

IHA: Concepción y diseño del estudio, diseño experimental, análisis e interpretación de datos, redacción de la primera versión del manuscrito, revisión del manuscrito, gestión del financiamiento.

\section{Referencias bibliográficas}

Alvarado Ibarra M, Luis Álvarez Vera J, Anaya Cuéllar I, De La Peña Celaya A, García Fernández L, Hernández Ruiz E, Herrera Olivares W, Leyto Cruz F, Loera Fragoso et al. 2015. Primer Consenso Nacional de Mieloma Múltiple por Hematólogos del ISSSTE. artículo Espec Rev Hematol Mex. 16: 306-332.

Arce Hernández AA, Villaescusa Blanco R, Morera Barrios LM, Junco González Y, Merlín Linares JC, Ramón Rodríguez L, Hernández Padrón C. 2013. Distribution of types of paraproteins in a sample of patients with multiple myeloma. Rev Cuba hematol inmunol hemoter. 29(2): 183-188.

Caravaca Fontán F, Gutiérrez E, Delgado R, Praga M. 2017. Monoclonal gammopathies of renal significance. Nefrol English Ed. 37(5): 465-477.

Chang CC, Su MJ, Lee SJ, Tsai YH, Kuo LY, Lin IH, Huang HL, Yen TH, Chu FY. 2016. The immunotyping distribution of serum monoclonal paraprotein and environmental impact on multiple myeloma 
(MM) and monoclonal gammopathy of uncertain significance (MGUS) in Taiwan: A medical center-based experience. Asian Pacific J Cancer Prev. 17(1): 395-399.

Cueva Ayala P, Yépez Maldonado J, Tarupi Montenegro W. 2019. Epidemiología del Cáncer en Quito. 16th ed. Quito. Ecuador: Editorial UTE.

Delgado MF. 2019. Utilidad del ensayo de cadenas livianas libres en suero en el manejo de gammapatías monoclonales: diagnóstico, pronóstico y monitoreo. Rev Médica del Uruguay. 30(1): 65-75.

García García P, Enciso Alvarez K, Diaz Espada F, Vargas Nuñez JA, Moraru M, Yebra-Bango M. 2015. Gammapatías biclonales: estudio retrospectivo de 47 pacientes. Rev Clin Esp. 215(1): 18-24.

Heaney JL, Campbell JP, Griffin AE, Birtwistle J, Shemar M, Child JA, Gregory WM, Cairns DA, Morgan G, Jackson G, et al. 2017. Diagnosis and monitoring for light chain only and oligosecretory myeloma using serum free light chain tests. Br J Haematol. 178(2): 220-230. Jenner E. 2014. Serum free light chains in clinical laboratory diagnostics. Clin Chim Acta. 427: 15-20.

Laribi K, Alani M, Lemaire P. 2018. IgM k multiple myeloma with monoclonal surface immunoglobulin expression. Int J Hematol. 108(1): 3-4.

Leone PE, Cabrera-Andrade A, García-Cárdenas JM, González DA, Guevara-Ramírez P, LópezCortés A, Zambrano AK, Paz-y-Miño C. 2017. Ancestry study in Ecuadorian population with multiple myeloma. Forensic Sci Int Genet Suppl Ser. 6: 435-436.

Marco P. 2014. El laboratorio en el diagnóstico de las gammapatías monoclonales. Dep Bioquímica Clínica INFIBIOC Fac Farm y Bioquímica UBACentro Hematol Pavlov. 18: Numero extraordinario 9-11.

McCudden CR, Mathews SP, Hainsworth SA, Chapman JF, Hammett-Stabler CA, Willis MS, Grenache DG. 2008. Performance comparison of capillary and agarose gel electrophoresis for the identification and characterization of monoclonal immunoglobulins. Am J Clin Pathol. 129(3): 451-458.

Montesdeoca Narváez MB. 2015. Estado del mieloma múltiple, diagnóstico clínico, medicina de laboratorio e identificación de alteraciones genéticas asociadas con la patogénesis en el Hospital Carlos Andrade Marín en un periodo de tiempo comprendido entre 1986 a 2012.

Morrison T, Booth RA, Hauff K, Berardi P, Visram A. 2019. Laboratory assessment of multiple myeloma. 1st ed. Elsevier Inc. Murata K, Mccash S, Landgren CO. 2019. 80 - Monoclonal Gammopathies. In: Clinical Immunology. Principles and Practice. Fifth Edit. Elsevier Ltd. 10791093.

Osatinsky R, Desimone I, Garnek L. 2004. Incidencia de patente oligoclonal en población adulta aparentemente sana. Rev Mex Patol Clínica. 51 (2): 90-92.

Peña C, Ortiz M, Voisin J, Peralta A, Balboa V, Delgado F. 2018. Sensibilidad diagnóstica de electroforesis de proteínas y cadenas livianas libres séricas en gammapatías monoclonales. Rev Med Chil. 146(1): 64-67.

Pizarro R, Samanez C, Cartolin M, Delgado F. 2016. Efecto de la medición de cadenas ligeras libres en suero en el diagnóstico de gammapatías monoclonales; experiencia en Perú. Rev Hematol Mex. 17(2): 99-106.

Rajkumar SV. 2018. Multiple myeloma: 2018 update on diagnosis, risk-stratification, and management. Am J Hematol. 93(8): 1091-1110. 
Rajkumar SV, Dimopoulos MA, Palumbo A, Blade J, Merlini G, Mateos MV, Kumar S, Hillengass J, Kastritis E, Richardson P, et al. 2014. International Myeloma Working Group updated criteria for the diagnosis of multiple myeloma. Lancet Oncol. 15(12): 538-548.

Rincón N. 2016. Neoplasias hematológicas: un mundo aún por descubrir Hematologic neoplasms: a world yet to be discovered Hospital General de Medellín. Med Lab. 22(3-4): 109-110.

Seth S, Zanwar S, Vu L, Kapoor P. 2020. Monoclonal Gammopathy of Undetermined Significance: Current Concepts and Future Prospects. Curr Hematol Malig Rep. 15(2): 45-55.

Vázquez AP, Palma AZ. 2015. Utilidad de pruebas de laboratorio en el diagnóstico de mieloma múltiple. Rev Latinoam Patol Clínica. 62(1): 55-62. 